\title{
Linx
}

LinX Revue des linguistes de l'université Paris X Nanterre

$62-63 \mid 2010$

Entre syntaxe et discours. Éclairages

épistémologiques et descriptions linguistiques

\section{Les pronoms « neutres » et leur référence à des procès en français parlé}

\section{Laure Anne Johnsen}

\section{(2) OpenEdition \\ Journals}

Édition électronique

URL : http://journals.openedition.org/linx/1382

DOI : $10.4000 /$ linx.1382

ISSN : 2118-9692

Éditeur

Presses universitaires de Paris Nanterre

Édition imprimée

Date de publication : 1 juillet 2010

Pagination : 153-178

ISSN : 0246-8743

Référence électronique

Laure Anne Johnsen, «Les pronoms « neutres » et leur référence à des procès en français parlé », Linx [En ligne], 62-63 | 2010, mis en ligne le 04 décembre 2013, consulté le 01 mai 2019. URL : http:// journals.openedition.org/linx/1382 ; DOI : 10.4000/linx.1382 


\title{
Les pronoms « neutres" et leur référence à des procès en français parlé
}

\author{
Laure Anne Johnsen \\ Universités de Neuchâtel et de Fribourg \\ laure.johnsen@unifr.ch
}

\section{Introduction}

Cet article ${ }^{1}$ se propose de fournir un éclairage sur le fonctionnement anaphorique des pronoms dits «neutres » dans des extraits de français parlé, lorsqu'ils réfèrent à des procès au sens large, i.e. des états de choses typiquement dénotés par des constructions verbales, qu'il s'agisse d'événements, de faits, de propositions, d'états ou encore de situations :

(1) $[\ldots]$ à cinq heures de l'après-midi, faut te mettre dans la tente parce que là, tu as les moustiques qui viennent partout. Donc ça c'est l'horreur. (PFC, Genève)

En (1), le pronom $c ̧ a$ redoublé du clitique $c$ ' réfère à un objet, vraisemblablement la situation ou l'état résultant de l'invasion des moustiques (dans la jungle amazonienne, à l'heure en question) : en effet, c'est à ce référent que le locuteur attribue la propriété d'être l'horreur'. On peut traiter le procédé référentiel en termes d'anaphore au sens traditionnel, par le fait que le pronom renvoie à un objet rendu disponible par le contexte verbal qui précède. Néanmoins, ce genre d'exemple diffère des cas typiques

\footnotetext{
${ }^{1}$ Cette étude s’inscrit dans le cadre du projet FNS n 100012-122251 (« Syndèse et asyndèse dans les routines paratactiques du français »), hébergé par l’Université de Neuchâtel.
} 
d'anaphore présentés dans les grammaires sur au moins trois aspects : i) le référent visé n'est pas une entité préalablement catégorisée ; ii) le « déclencheur d'antécédent» (Cornish, 1999) (autrement dit le «support segmental » qui a rendu le référent disponible), pour autant qu'il soit identifiable, consiste en au moins une construction verbale; iii) le pronom neutre maintient la non catégorisation du procès dans le segment anaphorique. Ces caractéristiques rendent ardue une description linguistique du procédé référentiel à l'œuvre. A défaut d'y apporter une solution, je me propose de soulever quelques problèmes liés à ces cas de figure et de suggérer quelques pistes de réflexion, en explorant les étapes allant de l'expression d'une (ou plusieurs) construction(s) verbale(s), autrement dit, de la dimension syntaxique, au pointage, via un pronom neutre, de son contenu au niveau discursif.

Dans les pages qui suivent, je commencerai par présenter les données analysées ( $(1)$ et par délimiter l'objet d'étude $(\mathbb{\$} 2)$. Je préciserai ensuite ce que recouvre la notion de pronom neutre $(\$ 3)$, et présenterai la conception du discours et de la référence que j’adopte (\$4). Je me pencherai sur le type d'objets-de-discours concerné ici, à savoir les procès, en inventoriant les typologies existantes de cette classe $(\mathbb{\$} 5$.). Ces outils théoriques seront confrontés à des données attestées de français parlé (\$ 6.1.-\$ 6.2.). On verra que ces typologies d'inspiration logique et philosophique présentent certaines limites face aux exemples attestés de l'oral et que dans de nombreux de cas, le procédé référentiel à l'œuvre manifeste une certaine sous-spécification (\$ 6.3.). On tentera néanmoins de distinguer différents cas de figure selon la manière dont est élaboré un procès, et l'on s'interrogera à cet égard sur le statut d'objet-de-discours : à partir de quand et selon quelles modalités «y a-t-il » objet-de-discours ( $($ 7.1.- $\$$ 7.2.) ? Quelques pistes seront évoquées par le recours aux notions d'activation et de validité des objets-de-discours en mémoire discursive (\$ 7.3.-7.4.).

\section{Les données analysées}

Les données d'oral exploitées ici sont essentiellement tirées de conversations libres ou d'entretiens semi-dirigés du corpus PFC ${ }^{2}$ (Durand et al., 2002, 2009), enregistrés dans les années 2000. Le corpus PFC est en cours de constitution et vise à couvrir une trentaine de lieux d'enquête de l'espace francophone international. Quelques exemples proviennent en outre du corpus CTFP ${ }^{3}$ (Blanche-Benveniste $e t$ al. 2002), qui rassemble trente-six extraits de conversation généralement semi-dirigée de locuteurs français, dont les enregistrements relativement anciens (date rarement communiquée) ont été transcrits dans les années 1990. Dans cet article, les exemples ont été reproduits avec les conventions de transcription des corpus d'origine. Il est à noter que la présente étude ne cherche pas à fournir des résultats quantitatifs ${ }^{4}$, mais

\footnotetext{
2 Phonologie du français contemporain. Une dizaine de minutes de chaque enregistrement est accessible sur le site http://www.projet-pfc.net, accompagnée d'une transcription orthographique.

${ }^{3}$ Choix de textes de français parlé.

${ }^{4}$ A cet égard, des indications chiffrées sur la répartition des pronoms seraient très difficiles à fournir, les pronoms en question ne référant pas toujours à des procès et ayant des homonymes (cf. le pronom personnel, le pronom neutre, le déterminant). Par ailleurs, les corpus retenus ici ne sont ni échantillonnés ni équilibrés de manière à pouvoir mener des analyses statistiques représentatives.
} 
elle s'efforce plutôt, à travers une démarche empirique à visée qualitative, d'attirer l'attention sur des faits linguistiques régulièrement attestés tout en dégageant les problèmes de description que ceux-ci manifestent.

\section{Délimitation de l'objet d'étude}

Mon attention porte sur la référence pronominale à des procès, autrement dit, à des objets prototypiquement formés sur la base d'informations issues de constructions verbales (désormais $\mathrm{CV}$ ), qu'on peut paraphraser au moyen d'une «proposition ». Un procès peut également être porté à la connaissance des interlocuteurs par d'autres moyens, comme des percepts situationnels (cas traditionnel de deixis, e.g., un locuteur aperçoit un passant traverser la route en courant et s'exclame : c'est dangereux.). Cela dit, je laisserai de côté ces cas de deixis, n'ayant accès qu'aux traces segmentales des corpus étudiés ${ }^{5}$. Je ne m'intéresserai pas non plus aux cas où le procès a été préalablement activé et catégorisé en mémoire par le biais d'un SN (de type nominalisation), ce qui semble conférer à l'entité un statut cognitif différent (plus élevé) (Gundel et al., 2003, p. 284).

Certains travaux se sont penchés sur la question, mais avec des points de vue très divers. De fait, la délimitation du problème est rarement co-extensive d'une étude à l'autre, ce que ne manquent pas de montrer les différentes étiquettes choisies, mettant tantôt l'accent sur l'antécédent (pronoms phrastiques < Amsili et al., 2007), tantôt sur l'anaphorique (complex anaphor < Consten et al., 2007), tantôt sur le référent impliqué (extended reference ou reference to fact $<$ Halliday \& Hasan, 1976; situational anaphora, < Fraurud, 1992 ; abstract object anaphora < Asher, 1993 ; propositional anaphora ; Cornish, 1991), tantôt enfin sur l'opération référentielle ou cognitive en jeu (discourse deixis < Webber, 1991 ; anaphore conceptuelle $<$ Descombes Dénervaud \& Jespersen, 1992). Afin de ne pas biaiser le point de vue adopté, je renoncerai à user d'une étiquette prédéfinie en privilégiant la prise en compte globale du processus à l'œuvre, à savoir, le procédé de référence pronominale à un procès.

\section{Les pronoms « neutres »}

Par " pronom neutre », j'entends un pronom qui n'implique pas de catégorisation nominale de son référent ${ }^{6}$, par opposition au clitique de $3^{\mathrm{e}}$ personne :

(2) E: Tu as interêt d'y aller avec un guide dans ces endroits-là. [...] JD: Après tu as, justement tu as différents moyens, nous, nous, on a fait ça comme ça sur une petite pirogue. Bon aussi, ça coûtait moins cher donc ça nous arrangeait bien. (PFC, Genève)

\footnotetext{
${ }^{5}$ Les linguistes sont souvent contraints à travailler sur des données empiriques qui ne sont que des traces fragmentaires (graphiques et acoustiques) des activités discursives effectives, alors qu'il y a des raisons de penser que le discours constitue un «complexe pluri-codique » (Groupe de Fribourg, à par, p. 21).

${ }^{6}$ Ce qui ne veut pas dire que le référent soit non catégorisé : celui-ci peut avoir été préalablement catégorisé (cf. le poisson, ça pue). Un pronom neutre ne dit rien en soi quant à la catégorisation de son objet, il est donc non-marqué de ce point de vue.
} 
(3) $[\ldots]$ elle coûtait moins cher donc ça nous arrangeait bien (exemple modifié)

Dans l'exemple modifié en (3), le pronom elle indiquerait que son référent est classifié, ici par exemple au moyen de l'étiquette lexicale disponible pirogue (fém.). A l'inverse, un pronom neutre ne donne aucune indication à cet égard. "Neutre» ne qualifie donc pas le genre grammatical, puisque ce type de pronom s'accorde évidemment au masculin, qui sert de genre non-marqué en français.

Pour une illustration supplémentaire, comparer l'homonymie entre un pronom catégorisant (4) et un pronom neutre (5), dans les deux cas régimes directs du verbe :

(4) Mais té, tu vois si j'étais pas là ce soir-là, $(\mathrm{XX})$, il brûlait ma maison, cette case, quoi. Et ça là, je mets ma main à couper. Parce qu'il a essayé de faire, et le pire là, c'est que je le croyais pas. Je le croyais pas capable (PFC, Ile de la Réunion)

(5) je, je pense que j’ai été sévère avec mes enfants, mais pas, étriquée pas étroite à ce point-là. Faut quand même pas exagérer hein. Bon. J'ai un mari qui m'a beaucoup ouvert, il faut que je le dise parce que sinon il sera pas content. (PFC, Roanne)

En (4), le réfère, du moins dans sa seconde occurrence, à un individu préalablement activé (coréférant au il qui précède), présenté comme un être humain, par définition classifié, et sémantiquement conforme au type d'argument $x$ sélectionné par le prédicat 'ne pas croire $x$ capable'. En (5) au contraire, le pronom le réfère au contenu activé par la CV J'ai un mari qui m'a beaucoup ouvert, autrement dit, un procès. Les procès ne possédant pas a priori de "nom en propre » (Kleiber, 1994a, p. 24), il n'est pas étonnant que l'on recoure volontiers aux pronoms neutres pour y référer.

Dans cette catégorie de pronoms, on trouve les clitiques ce (nominatif), le (accusatif), en et $y$ (cas obliques), et les toniques cela, ceci, le pronom ça pouvant relever des deux types ${ }^{7}$. Les premières observations sur les données livrées par le corpus PFC révèlent que les pronoms les plus productifs dans la référence aux procès sont ce et ça. $C e$ et ça clitiques ne fonctionnent qu'en position de sujet tandis que ça tonique peut occuper la place d'un sujet détaché ou d'un objet direct, et peut compléter une préposition (pour ça, à ça, de ça, etc.). Les autres pronoms neutres sont moins attestés pour la référence à des procès dans mon corpus ${ }^{8}$. Une raison pourrait être qu'à la différence de ce et ça qui sont toujours non-marqués quant à la catégorisation du référent, le/en/y réalisent aussi bien des emplois catégorisants ${ }^{9}$ (cf. l'exemple 4) que

\footnotetext{
${ }^{7}$ Un ça tonique peut être redoublé d'un ça clitique : puis j'ai passé l'agrégation externe d'économie gestion, euh, à Paris, j'ai été affectée d'abord euh, au, à Melun Sénart, dans un I.U.T. eub, 'techniques de commercialisation', dono ça ça a duré trois ans (PFC, Biarritz)

${ }^{8}$ Le clitique en apparait plus souvent dans des locutions où le pronom a perdu sa valeur référentielle (je m'en sortais pas, $j$ 'en sais rien, j'en avais marre) ou alors lorsqu'il réfère a des objets catégorisés. Le pronom $y$ réfère également à des objets ou à des lieux catégorisés et apparait destitué de sa valeur référentielle dans les constructions présentatives (ily a X).

${ }^{9}$ C'est leur emploi le plus fréquent, selon Goosse \& Grevisse $(2007, \$$ 678) : « Le plus souvent, en et $y$ désigne des choses ou des animaux: Les jolies fleurs! Donne m'en!; J'ai recueilli un chien et je m'y suis attaché.»
} 
non catégorisants, ce qui peut favoriser des ambiguités d'interprétation ${ }^{10}$. Cela dit, ils ne sont pas pour autant absents du corpus (cf. l'exemple 5 pour le). En voici un aperçu :

(6) Bon moi j'ai travaillé mais vous me direz euh. Dans des conditions euh agréables euh j’avais mes horaires je me rapportais du travail à la maison. Tout ça était tellement souple que j'étais quand même beaucoup avec les enfants. Mais euh je sais pas si ils s'en sont tellement aperçus hein. (PFC, Paris Centre ville)

(7) E1 : Et tu as jamais envisagé de partir de Marseille, en fait?

DG1: Non jamais, ça s'est jamais présenté. J'aurais pu pens/ y penser lorsque euh je faisais mes études, parce que euh, c’était pas évident de s'installer sur Marseille (PFC, Marseille)

En ce qui concerne ceci et cela désignateurs de procès, ils sont quasi-absents du corpus PFC. En effet, ils apparaissent essentiellement dans des locutions (ceci/ cela dit, ceci/cela mis à part, cela étant) ou en corrélation (je vous ferai ceci je vous ferai cela), où le phénomène de lexicalisation tend à affaiblir, voire à annuler leur valeur référentielle. A noter également que les rares occurrences trouvées en dehors des locutions proviennent essentiellement de locuteurs francophones d'Afrique ${ }^{11}$, comme dans l'exemple ci-dessous :

(8) Maintenant, les habitants de ce pays, on est tellement interpénétrés, que, aujourd'hui, euh, diviser le pays, ça ne va pas. C'est-à-dire, tu ne peux pas. Parce que il y a eu trop d'interpénétration, dans ce pays là, que il faut (bruit) que les gens, euh, reviennent à la sagesse, pour se dire que: 'on s'est trompé. On a échoué. et on repart sur un autre chemin'. Tant que les gens ne reconnaitront pas cela et qu'ils veulent persister dans leur, euh, bêtise, bon, on sera toujours dans, dans cette bêtise-là (PFC, Abidjan)

On peut supposer que le faible nombre d'occurrences de ceci, cela, mais aussi de $e n, y$ et dans une moindre mesure le, soit en lien avec le genre de corpus investigué, à savoir, de l'oral spontané ${ }^{12}$. Sans aller plus loin sur ces questions de distribution, signalons simplement à titre indicatif qu'une recherche de cela sur la base de données Frantext ${ }^{13}$ (textes littéraires, philosophiques, scientifiques et techniques), à l'exclusion des occurrences de cela dit, cela étant, cela mis à part, cela va sans dire, délivre un peu plus de 5800 résultats sur la centaine de textes du $21^{\mathrm{e}}$ siècle.

\footnotetext{
10 A noter aussi l'emploi du clitique neutre le pour les anaphores de prédicat, dont nous ne tenons pas compte ici. Sur ce sujet, je renvoie à Cornish (1991).

11 Pour le corpus PFC, je n'ai pas jugé utile de restreindre mon champ d'observation à la France métropolitaine ou à d'autres sous-catégories. S'il peut y avoir des différences (diatopiques, diastratiques, etc.) au niveau de la distribution des formes pronominales, mon intérêt porte davantage sur le processus de référence en jeu, à l'œuvre dans toutes les variétés de français.

12 Cela rappelle le fonctionnement des nominalisations lexicales : « [i] est probable que les nominalisations lexicales sont plus ou moins associées au registre écrit soigné » (Apothéloz, 1995a, p. 148).

13 http://www.frantext.fr
} 
Les pronoms neutres sont fortement sous-spécifiés et hormis l'indication du non marquage d'une classification nominale du référent, les indices sémantiques des marqueurs sont ténus. En ce qui concerne le, en, $y$, on associe généralement à leur nature clitique l'indication d'une continuité référentielle anaphorique (Cornish, 1991, voir aussi infra \6.5.). Quant à $c e / c ̧$, on pourrait évoquer les propriétés liées aux démonstratifs. Mais à $\mathrm{y}$ regarder de près, $c e / c ̧ a$ clitiques référant à des procès n'entrent en concurrence avec aucun clitique neutre non démonstratif en position de sujet (tel que it pour l'anglais, es pour l'allemand). Utilisés en quelque sorte par défaut dans cette position, est-ce qu'ils reflètent réellement les propriétés des démonstratifs ? La même question se pose pour le pronom ça tonique, qui ne commute avec aucun pronom tonique neutre non démonstratif ${ }^{14}$. Ces pronoms servant à «combler les trous » de déclinaisons défectives, il est légitime de se demander s'ils sont les meilleurs représentants des propriétés qu'on reconnait habituellement aux démonstratifs, telles que la token-réflexivité (le fait qu'une occurrence s'interprète à travers son contexte d'apparition), la capacité à attirer l'attention sur un référent, ou la présentation d'un aspect nouveau d'un référent ${ }^{15}$.

\section{Le discours : une approche cognitive}

Le développement d'une approche cognitive du discours, dès les années 197080, me semble découler, entre autres, de la reconnaissance de certaines limites liées à la notion traditionnelle de référence : une première limite a trait à la description de la référence issue d'une tradition philosophique et à la nature des objets auxquels on fait référence $(\$ 4.1$.$) ; une autre limite concerne le traitement des expressions anaphoriques$ en linguistique textuelle ( $\$ 4.2$.$) .$

\subsection{La nature des référents}

Dans une perspective philosophique, une expression référentielle est censée dénoter un objet du monde réel, auquel on accède à partir du sens de cette expression, qui indique le mode de donation de l'objet, i.e., la manière dont est présenté l'objet. Cette approche s'attache ainsi à mesurer la conformité de la langue par rapport au monde. Un problème surgit alors pour des expressions n'ayant pas de représentants existant dans le monde qui nous entoure, telles que par exemple les SN le nombre entier le plus grand, la licorne de mon voisin, etc. Pour Frege (1892[1971], p. 89) et ses continuateurs, ces expressions n'ont tout simplement pas de dénotation; elles n'ont qu'un sens. D'un point de vue linguistique néanmoins, cette position n'est pas tenable, puisque la propriété référentielle des expressions linguistiques repose sur un «axiome d'existence » (Kleiber, 1997, p. 10) : «Tout ce à quoi on réfère doit exister » (Searle, 1972, p. 121). La question porte alors plutôt sur la nature de cette existence. Une solution, adoptée par certains, est de situer ces référents dans des mondes alternatifs, autrement dit, des mondes possibles (Kripke 1959). Ainsi, une expression pourrait référer à un objet du

\footnotetext{
${ }^{14}$ Cf. Je pense à ça (cela, ceci) vs. Je pense à celui-ci / hii; celle-ci / elle, etc. A noter également que les clitiques neutres le, en, $y$, n'ont pas la même distribution que le tonique ça avec lequel ils peuvent coexister : j'y pense, à ça; ça je le vois bien, etc.

15 Voir à ce sujet les articles de Cornish et Buscail dans ce volume.
} 
monde réel ou à un objet d'un monde imaginaire (Kleiber, 1997, p. 11). L'approche cognitive propose une autre solution, comme on le verra infra (\$4.3.).

\subsection{Le traitement des expressions anaphoriques en linguistique textuelle}

Depuis l'émergence de la linguistique textuelle, les procédés anaphoriques ont été intégrés à l'étude des phénomènes de cohérence et de progression thématique à l'œuvre dans un texte, dont on peut tenir pour représentatif l'ouvrage d'Halliday \& Hasan, Cohesion in English (1976). L'anaphore, de ce point de vue, est décrite comme un processus de dépendance sémantique d'une expression, dite anaphorique, à un antécédent à récupérer en amont dans le texte, ou plus simplement, comme un phénomène de reprise : «l'anaphore se définit traditionnellement comme toute reprise d'un élément antérieur dans un texte » (Riegel et al., 2009, p. 1029), comme en témoignent des exemples du genre :

(9) Depuis trois jours la seule distraction de Mme de Rênal avait été de tailler et de faire faire en toute hâte par Elisa une robe d'été, d'une jolie petite étoffe fort à la mode. A peine cette robe put-elle être terminée quelques instants après l'arrivée de Julien ; Mme de Rênal la mit aussitôt. (Stendhal < ibid.)

Cette conception de l'anaphore, largement répandue dans les grammaires et en linguistique textuelle, mais aussi en intelligence artificielle et dans certains travaux en psycholinguistique, a pour souci principal l'identification d'un antécédent en amont dans le texte, et cela ne va pas sans poser certains problèmes, comme le souligne Kleiber (1994a, p. 38). La recherche d'un antécédent paraît notamment peu vraisemblable dans la chaine parlée, puisque la trace « textuelle » d'un événement communicatif est forcément de court terme (Cornish 2010, p. 210). Aussi, l'interprétation anaphorique ne semble pas passer par une mémorisation littérale des énoncés produits. Une vision textualiste de l'anaphore se heurte également à des phénomènes de «discordances morphosyntaxiques, sémantiques, et/ou référentielles » ([Reichler]-Béguelin, 1988, p. 18) :

(10) La foule a bravé le froid pour venir accueillir leur futur président (internet, légende d'une photo)

Ce genre d'anaphore indirecte implique l'inférence d'une classe à partir d'un individu collectif. Cet exemple ne saurait trouver une explication satisfaisante uniquement en termes de reprise ou de dépendance textuelle. L'interprétation anaphorique fait intervenir des facteurs opérant à différents niveaux de l'analyse linguistique (lexicosémantiques, pragmatiques, cognitifs, etc.)

\subsection{Un modèle du discours}

Les questionnements sur la nature des référents et ceux soulevés par une approche textuelle des procédés anaphoriques trouvent des éléments de réponse à travers la conception du discours que proposent des linguistes ou psycholinguistes dans des travaux d'inspiration cognitiviste au cours des années 1970-80. En effet, selon ces chercheurs, les interlocuteurs construisent un modèle du discours à partir des éléments textuels et de la communication à l'œuvre : 
(...) let us say that a TEXT is a set of instructions from a speaker to a hearer on how to construct a particular DISCOURSE-MODEL. The model will contain DISCOURSE ENTITIES, ATTRIBUTES, and LINKS between entities. A discourse entity is a discourse-model object, akin to Karttunen's (1971) DISCOURSE REFERENT. (Prince, 1981, p. 235)

Ce type de modèle mental (Johnson-Laird, 1983) constitue ainsi une représentation cognitive des informations engrangées au fil du discours. Les objets dont on parle sont donc également des représentations cognitives qu'on a pris pour habitude d'appeler à cet effet objets-de-discours. Cette approche se distingue de celle qui recourt aux mondes possibles : les objets sont ici le fruit du discours construit et co-existent, non pas en tant que realia du monde ou référents d'autres mondes, mais au sein d'un modèle discursif.

Le cadre d'analyse que j'utilise, développé par le Groupe de Fribourg dans sa Grammaire de la période (à par.), mais déjà, entre autres, dans [Reichler-]Béguelin (1988), Berrendonner (1990) et (1994), Apothéloz, (1995a) et (1995b), s’inspire de ces travaux cognitivistes, à travers son approche « constructiviste » du discours. En effet, celui-ci a pour fonction l'élaboration d'une mémoire discursive (désormais M) entre les interlocuteurs, c'est-à-dire la construction d'un ensemble évolutif de représentations publiquement partagées entre eux. La mémoire discursive se caractérise entre autres par le fait d'être constamment alimentée non seulement par des données textuelles, mais aussi par des percepts, des savoirs encyclopédiques manifestes, des indices gestuels, iconiques, etc. En outre, la manière dont les états de $M$ évoluent au fil du discours est fortement conditionnée par les opérations d’inférence sur les représentations cognitives et par la structure pragma-syntaxique du discours, i.e. la combinaison des énonciations entre elles, le type d'actions communicatives qu'elles produisent, etc.

\subsection{L'opération référentielle}

Ce recours à la notion de mémoire discursive permet de décrire les procédés référentiels comme des pointages ${ }^{16}$ linguistiques sur des objets-de-discours, quel que soit le mode d'introduction de ceux-ci en M (verbal, perceptif - procédé respectivement anaphorique ou déictique, au sens traditionnel de ces termes ${ }^{17}-$ ou autre $)^{18}$. Dans le cas de l'anaphore discursive, c'est la désignation d'un objet que l'activité discursive est supposée avoir rendu disponible en $\mathrm{M}$ qui importe, plutôt que la récupération d'un antécédent textuel. L'interprétation anaphorique n'est dès lors plus une affaire de mémorisation littérale du texte en amont, mais une question de réactivation d'une information stockée dans $\mathrm{M}$ au fil du discours, dont les modalités sont notamment déterminées par des facteurs sémantiques, pragmatiques et cognitifs.

Pour ce qui concerne la référence aux procès, la situation est un peu différente de celle où un anaphorique renvoie à un objet individué préalablement catégorisé dans

\footnotetext{
16 Par analogie aux pointages gestuels.

17 Pour une alternative à cette opposition traditionnelle, voir les articles de Cornish et de Buscail dans ce volume.

${ }^{18}$ Les représentations en $\mathrm{M}$ sont en fait sémiotiquement indifférenciées, puisque indépendantes de la modalité par laquelle elles ont été introduites (Groupe de Fribourg, à par., p. 23).
} 
M (cf. l'exemple 9). En effet, un procès exprimé par prédication au moyen d'une CV ne constitue pas un objet catégorisé dans $M$ et nommable via une dénomination particulière :

Les actions, événements, etc., ne disposent pas de noms en propre. Ils n'appartiennent pas à une classe référentielle dont les individus portent le même nom. Opposés aux entités classifiées, qui sont en quelque sorte des « choses nommées », ils ne sont que des choses. (Kleiber, 1994a : p. 24)

Un pronom neutre permet, tout en condensant les informations-supports de la $\mathrm{CV}$, de référer à un procès dans $\mathrm{M}$, sans pour autant le catégoriser, par opposition aux nominalisations lexicales anaphoriques (Apothéloz 1995a : p. 144). Un pronom neutre est également apte à synthétiser des informations-supports provenant de plusieurs $\mathrm{CV}$, informations formant ainsi un tout complexe (ibid.: p. 147), comme on en verra des exemples infra (19 et 20).

\section{Nature et typologies des procès}

Les procès, on l'a vu, sont un type d'entités que dénotent notamment les CV. Malgré l'absence de dénomination conventionnelle qui caractérise notamment ce genre d'objets, toute une tradition logico-philosophique range ceux-là sous des étiquettes telles qu'événement, action, activité, état, situation, proposition, fait, etc. Diverses typologies ont été avancées à des fins de hiérarchisation de la classe, distinguant d'une part les divers types de procès entre eux, d'autre part les procès des autres types d'objets.

\subsection{Evénements, faits et propositions}

D'après Vendler (1967), on peut distinguer parmi la dénotation des sentential nominals ${ }^{19}$ en anglais deux grands types sémantiques, selon que les nominals en question sont admis par tel ou tel prédicat, appelé container: les tight containers (tels que to occur, take place, begin, last, etc.) se combinent avec des arguments de type événements (actions, processus) dénotés par des perfective nominals, et les loose containers (to be unlikeely/certain, surprise, cause, etc.) avec des faits, dénotés par des imperfective nominals. Par la suite, Vendler (1970) juge nécessaire d'ajouter une troisième catégorie, qu'il nomme propositions, fonctionnant comme arguments de containers tels que to believe ou to deny. Cette distinction entre événements, faits et propositions connaittra une fortune considérable en linguistique.

\subsection{Les différents ordres de Lyons (1977)}

La distinction entre les éléments qui peuvent «survenir» et les objets qui ne manifestent pas de caractéristiques spatio-temporelles se retrouve en partie chez Lyons (1977). Celui-ci suggère de classer les entités en général en différents ordres: le premier ordre concerne les objets physiques (personnes, animaux, choses), dont on dit qu'ils

${ }^{19}$ C'est-à-dire des expressions "phrastiques » pouvant fonctionner comme arguments de verbes, telles que that John died, John dying, ou John's death. 
'existent', et qui possèdent des propriétés perceptuelles qui les rendent spatiotemporellement situables et observables. Les entités du deuxième ordre 'surviennent' ou 'ont lieu': il s'agit d'événements, processus et états de choses, eux aussi localisés dans l'espace-temps. Enfin, à la différence des autres types, les entités du troisième ordre n'ont pas de localisation spatio-temporelle et ne sont pas observables : elles sont susceptibles d'être affirmées ou niées, d'être jugées vraies ou fausses. Lyons y range les objets d'attitudes propositionnelles (croyance, espérance, jugement, etc. ${ }^{20}$ ).

\subsection{Une échelle d'immanence mondaine}

Asher (1993) se propose d'affiner la typologie de Vendler (1967). Les prédicats étudiés sélectionnent des types ontologiques distincts à situer sur une échelle d'immanence mondaine (world immanence spectrum) : les entités à caractère événementiel (événements, états, activités, accomplissements, achèvements) se placent à l'extrémité « concrète » de l'échelle en vertu de leurs propriétés spatio-temporelles, de leur efficacité causale et de leur contingence. A l'opposé, les entités de type 'propositionnel' ne possèdent pas ces caractéristiques : elles existent indépendamment de l'espace-temps. Les entités « factuelles » (faits, possibilités, situations, états de choses) se situent entre les deux pôles d'immanence, manifestant des propriétés tantôt propres aux événements, tantôt propres aux propositions. Cette échelle indique donc quelles entités sont plus ou moins bien établies dans le monde en fonction de la manière dont elles interagissent avec les autres entités dans l'espace-temps, autrement dit, ces distinctions reposent sur des critères relevant de la métaphysique de la langue naturelle (ibid., p. 30).

\subsection{Remarques sur les typologies proposées}

Dans les travaux évoqués, on constate que les paramètres typologiques sont des critères observables dans le monde : propriétés perceptuelles, ancrage spatio-temporel, relation de causalité (un procès peut être la cause de quelque chose), etc. L'approche est donc essentiellement vériconditionnelle dans le sens où l'on mesure si la langue est conforme aux données observables et comment elle s'adapte au monde : l'intérêt est d'étudier la manière dont les distinctions ontologiques naturelles se reflètent dans la langue. La démarche que je propose de suivre est inverse : elle consiste à observer ce que les faits linguistiques nous révèlent sur la manière dont les locuteurs se représentent le monde.

\section{La référence anaphorique aux procès dans un corpus de français parlé}

\subsection{Comment interpréter les pronoms sous-spécifiés?}

La pauvreté sémantique des pronoms neutres, dont tirent profit les pointages de procès, pose la question de leur traitement interprétatif. En effet, si le pronom neutre ne dit presque rien sur le référent, comment parvenir malgré tout à une interprétation?

\footnotetext{
${ }^{20}$ Cette typologie sera complétée par Dik (1989), qui intègre un ordre zéro comportant les prédicats, ainsi qu'un quatrième ordre d'entités regroupant les actes de langage (Apothéloz, 1995a, p. 158).
} 
Une solution proposée notamment par Cornish (1999, p. 69 sqq.), valable à ses yeux pour toute interprétation anaphorique, est, non pas de remonter jusqu'à l'antécédent, mais de s'appuyer sur le « segment indexical», autrement dit sur la prédication opérée sur l'expression référentielle. En effet, c'est ce qui est prédiqué du référent, bien plus que l'antécédent, qui détermine l'interprétation et la référence de l'élément anaphorique, par le biais de contraintes de sélection :

(11) Ca arrive souvent (<ibid., p. 77).

le prédicat arriver souvent s'applique à une situation ou à un état de choses (ibid., p. 78), condition que devra ainsi vérifier le référent de ça. Cette analyse rappelle le rôle des containers de Vendler (supra \5.1.).

\subsection{Cas prototypiques}

L'analyse linguistique qui s'appuie sur ces outils ainsi que sur les typologies présentées supra se montre relativement efficace sur un certain nombre de données :

(12) E: Ouais, ça il y a aussi, il y a aussi justement le rapport avec euh, comme on dit souvent, et même en Valais à travers, moi je le vois avec la linguistique. Le rapport patois, avec la langue, comme on dit, le français le rapport du patois par rapport à la langue et, malgré tout, même les accents ont toujours, eu quelque chose de d'inférieur alors que c'est faux. C'est faux de penser ça, $<$ MS: Moi, $\mathrm{j} />$ les les patois et les langues intérieures sont plus $<$ MS: Hum hum. $>$ riches. (PFC, Genève)

Dans l'exemple (12), le segment indexical du pronom c' restreint son interprétation à un argument pouvant être jugé faux. De même, le référent de ça de l'énoncé suivant est contraint d'être un objet de pensée (penser ça). Selon les typologies proposées supra, le type d'objet pouvant correspondre à ces contraintes sémantiques est une proposition. En effet, on peut considérer que c'est une proposition du genre 'le patois et les accents régionaux sont inférieurs à la langue standard' qui fait l'objet des pointages au moyen des pronoms neutres. Autre cas d'école, déjà cité plus haut (exemple 8 supra, \3):

(13) $[\ldots]$ il faut (bruit) que les gens, euh, reviennent à la sagesse, pour se dire que on s'est trompé. On a échoué. Et on repart sur un autre chemin. Tant que les gens ne reconnaîtront pas cela et qu'ils veulent persister dans leur, euh, bêtise, bon, on sera toujours dans, dans cette bêtise-là (PFC, Abidjan)

Ici, le référent de cela doit vérifier la contrainte sémantique exprimée par le segment indexical, à savoir celle d'être quelque chose que les gens du pays sont censés reconnaître. En contexte, le référent compatible avec cette contrainte, i.e. l'objet devant être reconnu, peut être glosé par 'le fait de s'être trompé et le fait d'avoir échoué' : en effet, on reconnait un fait, et non un événement survenu ou encore l'objet d'un dire ou d'un jugement. Voyons enfin un cas de référence à un procès de type 'événement' :

(14) Et, on, on trouve une prairie et puis, le mauvais temps arrive, brouillard arrive, bon, ben très bien ça va être au poil, $\mathrm{pa} /$ on va bien voir comment c'est. Euh, on s'installe, on plante notre tente, ça dure, euh, une petite demi- 
heure, on s'installe, ben puis s/ on replie notre tente, puis le brouillard se lève, et, on pensait être tranquilles dans notre prairie, et en réalité on était dans la cour d'une ferme. (PFC, Biarritz)

Dans cet exemple, le prédicat 'durer une petite demi-heure' signale explicitement l'ancrage spatio-temporel de l'objet en question. La propriété de 'durer' s'applique donc à une entité que l'on peut ranger dans la catégorie des événements. Ces exemples s'accommodent relativement bien des éléments de classification proposés jusque-là. Si l'on adopte ces outils d'analyse, on aura sans doute remarqué que le procès "d'entrée » (tel qu'il est introduit par la CV en mémoire discursive), n’est pas forcément du même type que le procès auquel on réfère via le pronom. Cela se voit dans l'exemple (13), où les procès 'on s'est trompé' et 'on a échoué' sont d'abord introduits en tant que propositions (objets du verbe dire), tandis qu'ils sont «récupérés » par cela sous la forme de faits (conformément au segment indexical). A cet égard, Consten et al. (2007) établissent une analogie entre ce type d'anaphore et les anaphores du $1^{\text {er }}$ ordre de Lyons (1977) : l'anaphore indirecte fonctionnerait de la même manière à ces deux niveaux. Une anaphore indirecte est un procédé par lequel une expression vise un référent à inférer à partir d'un référent-support déjà établi dans $M$, en vertu d'une relation discursive entre eux (autrement dit, une anaphore associative au sens large du terme, cf. Cornish, 2001, p. 1). Dans le cas de la référence aux procès, on pourrait, comme pour les référents du $1^{\text {er }}$ ordre, inférer un type de procès à partir d'un autre type par ce processus. Pour Consten et al. (ibid., p. 93) néanmoins, s’il y a changement ontologique, celui-ci ne peut se faire que dans un sens sur l'échelle d'immanence d'Asher, à savoir du concret vers l'abstrait, ce dont les auteurs rendent compte par le concept d'ontology changing complexation:

(15) The Americans tried to invade the building but were forced back by shots from the top floor. This proves that the situation in Bagdad isn't under control yet. $(<$ ibid., p. 88)

Selon les auteurs, le procès initial en (15) a d'abord été introduit en tant qu'événement. Le pronom this réfère non pas à l'événement en question, mais, étant donné la prédication 'proves that ...', au procès envisagé comme un fait, autrement dit à un type d'entité plus abstrait. Selon leurs observations, l'inverse, e.g. le passage d'un fait à un événement, n'est pas attesté. Or, l'exemple (13) supra semble contredire cette position puisqu'on passerait de la dénotation de propositions au rappel d'un fait.

\subsection{Problèmes en suspens}

\subsubsection{Contextes prédicatifs non catégorisants}

Si les typologies présentées ci-dessus conviennent plutôt bien à l'analyse des exemples traités jusque-là, certaines données semblent plus réfractaires. En effet, certains prédicats associés aux pronoms ne paraissent pas sélectionner clairement un type d'argument:

(16) E2: Alors vous allez commenter le match, tous les deux? J: Oui euh. E2: Ça va vous rappeler votre jeunesse. (PFC, Roanne) 
En (16), qu'est-ce qui 'va rappeler aux interlocuteurs leur jeunesse'? On pourrait considérer qu'il s'agit de l'événement de commenter le match (un commentaire peut 'avoir lieu', il peut avoir une durée, un début et une fin), de la situation dans laquelle ils se trouvent alors qu'ils commentent le match, ou encore du fait de commenter le match... Le segment indexical semble ainsi laisser le procès « indéterminé » quant au type sémantique, au moment du renvoi anaphorique.

L'exemple (17) montre que l'indétermination ne concerne pas seulement le type de procès, mais aussi la portée que recouvre la référence du pronom :

(17) $\mathrm{BM}:[\ldots]$ tu regardes le marché de Chenôve il a une, tu vois, il a quand même une certaine ampleur alors que tu regardes le marché de Talant. E: Il y a un marché à Talant?

BM : Ouais, il y a un petit marché à Talant, mais c'est trois, tu vois, c'est trois, trois pauvres trucs euh, c'est. Il y a aucune vie quoi, c'est pas un, c'est pas un marché euh.

$\mathrm{E}: \mathbf{C}^{\prime}$ est bizarre hein quand même hein? (PFC, Dijon)

Est-ce la situation de contraste entre les deux marchés en question que le locuteur E trouve bizarre, l'état-de-choses qui en résulte, ou alors le fait que le marché de Talant soit misérable ? Comment délimiter l'extension référentielle du pronom ? C'est l'occasion ici de souligner les limites de la recherche d'un antécédent textuel.

Ces exemples montrent qu'il n'est pas intuitif d'associer un type sémantique de procès à un prédicat verbal. L’interprétation des énoncés se fonde bien sur la spécification apportée par le segment indexical, de même que sur l'état de $M$ et sur d'autres indices non forcément linguistiques, mais rien n'indique qu'elle présuppose une catégorisation du procès. Plutôt que de ranger les référents dans des catégories ontologiques, il semble que les interactants se satisfont sans trop de problème de la référence à des contenus de pensée occasionnels non pré-catégorisés et interprétés sur le vif à partir des indications contextuelles en présence.

\subsubsection{La question des transformations ontologiques}

Dans le même registre, on peut s'interroger sur la pertinence des transformations catégorielles postulées par Consten et al. (2007) dans leur théorie de l'ontology changing complexation. En témoigne cette séquence de pronoms neutres au fil de laquelle il n'est pas évident d'identifier des modifications référentielles distinctives :

(18) maintenant je crois qu'il y en a en France des columbariums mais à cette époque-là il y en avait qu'un - il y avait que celui-là - euh mais il y avait du monde hein mais jamais j'aurais cru qu'il y avait tant de monde qui se faisait brûler c'est drôle hein mais enfin ça tient moins de place je crois bien que ça va devenir obligatoire ça (CTFP, La fleuriste)

En recourant aux typologies existantes, on serait tenté de convoquer l'analyse suivante : activation en mémoire de la proposition (car objet d'une croyance, cf. j'aurais cru) 'il y a tant de monde qui se fait brûler'; passage au fait qu'un si grand nombre de personnes se fait brûler' ou au fait de 'se faire brûler' dont on prédique qu'il 'est drôle'; puis transition vers le processus de «conservation» des corps (ou le résultat du 
processus) que représente l'incinération, que la locutrice juge moins encombrant que d'autres moyens (ça tient moins de place). Enfin, le prédicat d'obligation ('devenir obligatoire') porte sur ce même processus. Par ailleurs l'énoncé ainsi produit active en mémoire une proposition ('se faire brûler va devenir obligatoire') qui fait l'objet d'une croyance de la part de la locutrice (je crois bien que). Le schéma ci-dessous résume ces étapes :

'tant de monde se fait brûler' proposition le fait qu'autant de monde se fait brûler / se faire

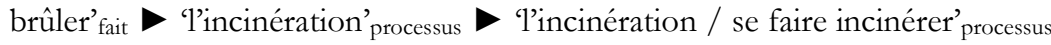

On peut se demander s'il y a vraiment lieu d'invoquer un processus de recatégorisation ontologique à chaque étape de pointage, alors qu'on a plutôt l'impression que la locutrice maintient grosso modo un même topique. Plutôt que de diagnostiquer des changements ontologiques (par ailleurs, pas toujours bien déterminés) dont seul un analyste pourrait avoir conscience au prix d'efforts d'abstraction considérables, il me semble plus pertinent de voir dans la «chaîne » de pronoms l'indice qu'on a affaire à une (co)référence approximative.

\subsubsection{Défaut d'indices linguistiques déterminants}

En fait, rien ne semble marquer formellement, dans la langue française, de telles distinctions. Par ailleurs, d'après les données typologiques de Corbett (1991) sur « l'accord neutre », aucune langue ne coderait des oppositions sémantiques de ce genre au moyen des pronoms (Fraurud, 1992). Au contraire, l'usage de pronoms sous-spécifiés et noncatégorisants, de même que la diversité des paraphrases possibles de ce genre d'objetde-discours, suggèrent plutôt une certaine indétermination catégorielle. Une solution, que retient Fraurud (1992), serait de ne conserver que deux grandes catégories, dont la limite est par ailleurs bien marquée dans les typologies existantes, à savoir, les événements et les faits. Une étude sur l'anglais (Gundel et al., 2003) montre que les événements dénotés par des CV sont directement mis dans le focus d'attention des interlocuteurs, et ainsi récupérables ultérieurement par un simple clitique (en l'occurrence it), tandis que les propositions ou les faits nécessitent l'usage d'un désignateur plus adapté (un démonstratif) en vue de la récupération de référents moins saillants dans le modèle discursif. Cela confirmerait, pour l'anglais, un statut particulier pour les événements, par rapport aux autres entités dès lors considérées comme plus « abstraites » (cf. l'échelle d'immanence $\int$ 5.3. supra).

Peut-être pertinente pour l'anglais, cette opposition ne paraît pas fonctionnelle en français. Il y a d'abord le fait qu'en position de sujet, les démonstratifs ce et ça n'entrent en concurrence avec aucun autre clitique pour la référence à des procès. Ensuite, on a vu que les clitiques objets $(l e, e n, y)$ sont tout à fait capables de référer à ce que la tradition nomme faits (ex. 6) ou propositions (ex. 5, 7). Quant aux pronoms toniques $(c a, c e l a, c e c i)$, ils ne paraissent pas non plus spécialisés dans la référence à l'un des types reconnus ${ }^{21}$. Il n'y a donc pas de critères linguistiques déterminants permettant d'attester de telles distinctions. Ce constat me permet d'éviter deux risques: d'une part, celui de transposer des catégories du monde (classification des

${ }^{21}$ Les exemples en (12), (13) et celui de la note 7 illustrent la référence à des « types » divers. 
phénomènes observables) telles quelles sur des faits linguistiques ; d'autre part, celui de transposer des catégories linguistiques sur le monde, tel que l'illustre ci-après le problème du recours à la notion de proposition.

\subsubsection{La notion de "proposition"}

La notion de proposition me parait problématique, lorsqu'elle est utilisée comme représentante d'un type sémantique que peut dénoter précisément... une proposition! En fait, il existe plusieurs conceptions de la proposition en vigueur dans le domaine linguistique, l'une, syntaxique, et l'autre, sémantique, héritée de la tradition logique. En effet, l'une dérive de l'autre, comme l'expliquent Riegel et al. (2009, p. 784-785) :

Le terme de proposition remonte aux grammaires logiques où il désignait toute construction minimale porteuse d'un jugement: l'association d'un sujet (ce dont on dit quelque chose) et d'un prédicat (ce que l'on dit du sujet). [...] Progressivement, la notion de proposition s'est confondue avec celle de phrase pour désigner l'unité syntaxique et prédicative combinant un sujet grammatical et un groupe verbal.

Il y a de quoi se méprendre, puisque les notions respectives sont simultanément en usage sur plusieurs terrains. On peut néanmoins désambiguïser un peu la situation en distinguant le contenu sémantique en tant que contenu propositionnel 22 , de l'unité syntaxique telle que la construction verbale. Les deux notions sont liées par le fait qu'une construction verbale exprime en principe un contenu propositionnel. Par ailleurs, il faut garder en tête que les deux notions sont des concepts métalinguistiques, que l'on se positionne du côté de la sémantique ou de la syntaxe.

Que recouvre alors la catégorie proposition dans les typologies présentées plus haut? Vraisemblablement pas une unité strictement syntaxique. Mais bizarrement, elle ne recouvre pas non plus la notion sémantique de contenu propositionnel. En effet, d'après ces typologies, tous les contenus propositionnels ne sont pas des propositions, puisque certains sont dits exprimer des faits, des événements, des situations et ainsi de suite. L'acception du terme proposition exploitée dans les typologies supra est donc plus restrictive que celle qui correspond à celle de contenu propositionnel. Elle correspond à un type de contenu propositionnel faisant l'objet d'une croyance, d'un dire ou d'une autre activité de l'esprit. Mais n'y a-t-il pas quelque chose de circulaire dans le fait qu'une construction verbale, par l'intermédiaire de son contenu propositionnel, puisse dénoter, entre autres, une proposition? Au côté de catégories mondaines telles que les événements et les faits, on ne comprend pas très bien ce que vient faire la catégorie métalinguistique de proposition: pourquoi la CV Roméo aime Juliette dénoterait un autre type de procès que la même CV dépendante d'un verbe de croyance tel que Jean croit que Roméo aime Juliette? Dans ce dernier énoncé, 'Jean' croit que l'état de choses dénoté est vrai mais il ne croit pas à une proposition! Cette dernière interprétation reviendrait à transposer une catégorie à l'origine linguistique sur un contenu de type

${ }^{22}$ Encore que la notion de "proposition» en logique ne corresponde pas tout à fait au contenu d'une proposition syntaxique : «les grammairiens de Port-Royal analysaient la phrase Dieu invisible a créé le monde visible en trois propositions : Dieu a créé le monde - Dieu est invisible - Le monde est visible » (Riegel et al., 2009, p. 785). 
mondain. Or, la dénotation d'un contenu propositionnel n'est pas conçue en mémoire comme une entité linguistique (sauf quand on se situe au niveau du métalangage ${ }^{23}$ ), mais comme un objet-de-discours mondain (Berrendonner 2008, p. 6).

Le recours à la notion de proposition dans ce genre de typologie peut conduire à de malencontreuses méprises. Pour cette raison, il me parait préférable de renoncer à son usage pour cette étude.

\subsubsection{Des contenus propositionnels pré-catégorisés?}

Certains travaux (Gundel et al., 2003, Consten et al., 2007) présupposent qu'un contenu propositionnel, autrement dit, la dénotation d'une $\mathrm{CV}$, est catégorisé dès le moment où il est simplement posé par un locuteur via la $\mathrm{CV}$, i.e. avant qu'il ne serve d'argument pour un prédicat dans le segment indexical (cf. supra \ 6.3.1). Cette position est également discutable au vu du manque d'indices linguistiques. En (19) ci-dessous, une fois la segmentation en $\mathrm{CV}$ réalisée ${ }^{24}$, il n'est pas évident de déterminer quelle sous-catégorie de procès chacune d'elles exprime :

(19) [Et alors donc euh, alors c'était super] [parce que, on était en fai/ en fait avec les Perez], [et on était donc], [ils avaient deux enfants avec eux, ou trois enfants, avec eux] [et puis nous on était quatre de notre côté,] [donc on était un bon groupe, ] [on partait le matin à neuf heures] [euh, pfff, on skiait euh, non pas tranquille quand même] [on skiait bien, jusqu'à midi et demie] [et là on s'arrêtait deux heures pour manger] [et puis, on repartait, deux trois heures l'après-midi]. [Mais, ça nous a permis de faire des grandes balades à travers les stations, qui se rejoignaient] [c'est quelque chose qu'on aurait pas osé faire tout seuls] [parce que, on savait pas minuter par exemple, les balades, et cætera] (PFC, Biarritz)

Dès la première $\mathrm{CV}$ posée, on rencontre une incertitude : comment décider si celle-là exprime, conformément aux typologies existantes, une situation, un état, un fait, une proposition? Idem pour les suivantes : la locutrice rapporte-t-elle des faits, des événements? Malgré d'éventuels indices livrés par les verbes (sens lexical, temps, modalité, aspect) ou les circonstants, il est difficile de déterminer si les procès ont la capacité de 'survenir', de 'surprendre', de 'causer', etc. Aussi me parait-il peu vraisemblable qu'un contenu posé sur le mode prédicatif présente le procès en question comme systématiquement pré-catégorisé en M. Cela ne signifie pas qu’il soit impossible, en tant qu'usager de la langue, d'employer intuitivement des désignateurs lexicaux «catégorisants» (e.g. Il nous est arrivé les événements suivants: [E1] [E2], etc.), ni de considérer tel ou tel procès comme une situation, un événement, une activité, etc.

23 Par exemple dans les cas de deixis textuelle, où un désignateur réfère à une entité linguistique du cotexte :

(X déclare) Ca, c'est un rbinocéros (et Y réplique) Un quoi ? Tu peux me l'épeler?

le pronom l' réfère à l'aspect orthographique et non au référent usuel du signe rbinocéros (Lyons, 1990, p. 289).

${ }^{24}$ La segmentation se montre problématique à certains égards, notamment dans le cas des enchâssements (P complétives, relatives, etc.), mais je laisse de côté ces questions qui n'ont pas d'incidences sur mon propos. 
C'est d'en faire des catégories théoriques préétablies et exclusives en vue de l'analyse que j'estime peu approprié à la description des faits linguistiques.

En somme, face au défaut d'exploitabilité des critères de classification proposés dans bien des contextes de français parlé spontané, il vaut mieux, pour notre approche de la référence pronominale aux procès, éviter de recourir à de telles typologies. Cellesci apparaissent empreintes d'une tradition logique et philosophique et ne parviennent pas à rendre compte des usages linguistiques effectifs de manière adéquate (Apothéloz, 1995a, p. 160). Par ailleurs, Asher (1993, p. 40) reconnait que sa typologie à caractère métaphysique peut présenter une certaine perméabilité entre les frontières établies, voire une certaine confusion entre les catégories :

It [the anaphora test] shows that there is an unexpected fluidity to the typing in the typology of abstract objects and suggests that at a more fundamental level, many distinct types of natural language metaphysics may be very similar, if not identical.

Cette remarque conforte l'idée que dans l'usage des locuteurs, les contenus propositionnels ne sont pas pré-catégorisés. C'est précisément le propre des procès de ne pas relever d'une classification nominale, à l'inverse des objets-de-discours possédant un niveau d'appellation de base (Rosch et al., 1976), ou plus généralement des objets «classifiés »(Cornish, 1991, p. 126, Kleiber, 1994a, p. 24). Il en va de même à mes yeux dans le cas d'une référence à un procès via un pronom neutre, par définition non catégorisant : il ne va pas de soi que le procès, bien que pris comme argument d'un prédicat - à cet égard, certes, davantage spécifié par ses nouveaux attributs - soit de fait classé dans une catégorie préétablie.

\section{De la construction verbale à l'objet-de-discours}

\subsection{Procès et agrégats}

L'exemple (19) supra montre aussi que les procès combinés entre eux par diverses relations de discours (temporelles, causales, etc., par ailleurs parfois implicites, cf. Corminboeuf, dans ce volume) et agglutinés au moyen de processus inférentiels, forment à leur tour des sortes de procès complexes que j’appelle agrégats. A la ligne 7 de (19) supra, la locutrice pointe explicitement sur l'une de ces «zones » en M, non pas activée par une seule construction verbale antérieure, mais inférée à partir de l'état de M sur la base d'indices verbaux (plusieurs CV qui précèdent) ou non (savoirs manifestes, indices suprasegmentaux, gestuels, autres percepts, etc.) : le référent de ça, en l'occurrence 'ce qui a permis à la locutrice et sa famille de skier sur différents domaines', c'est grosso modo 'la situation ou le fait ${ }^{25}$ d'avoir partagé leurs vacances avec

\footnotetext{
25 J'utilise ici «fait» ou «situation» dans leur sens conventionnel. En effet, ces notions ne me semblent pas incompatibles entre elles. Une analyse en sèmes à partir de contextes attestés pourrait révéler les relations que ces signifiés (et les autres) entretiennent entre eux dans l'usage des locuteurs du français.
} 
une autre famille vraisemblablement expérimentée et connaissant bien la région', et éventuellement 'd'avoir skié ensemble à un rythme relativement soutenu'. Ainsi, ce sont les informations-supports engrangées au fil des CV posées accompagnées d'autres types d'indices qui ont contribué à la construction inférentielle d'un tel agrégat que condense le pronom ça et qui impliquent en $\mathrm{M}$ toute une zone cognitive difficilement délimitable ${ }^{26}$.

La teneur référentielle de tels agrégats s'avère donc peu circonstanciée et l'on ne peut faire que des conjectures sur l'adéquation des gloses proposées. En voici encore un exemple, où en et $c^{\prime}$ réfèrent globalement au contenu des propos rapportés ici par le locuteur MS :

(20) (MS rapporte les propos de la mère de $\mathrm{E}$ )

MS: Et elle expliquait qu'elle voulait monter une association ou qu'elle, qu'elle était en train de la monter, pour pouvoir justement se faire payer, et avec l'argent, acheter du matériel, enfin qu'il y ait pas de euh, d'histoires de euh, d'argent euh, euh, <E: D'accord $>$ qui traîne quoi.

E: Ah ouais, bah, euh, peut-être, elle m'en a pas parlé. Mais, euh, c'est possible, parce que je sais qu'en ce moment elle donne des cours, à euh, à des copines. (PFC, Nantes)

\subsection{Le statut d'objet-de-discours}

Ce que l'on pourrait se demander à l'égard de ces agrégats, c'est s'ils préexistent à l'opération anaphorique en tant qu'entités discursives ou s'ils forment de telles unités uniquement dès qu'on y réfère. Autrement dit, les interlocuteurs procèdent-ils à la construction et à l'unification de certaines zones cognitives au fil des CV ou bien ce genre de condensé référentiel ne reçoit-il le statut d'objet qu'au moment du pointage référentiel ? La question ne se pose finalement pas seulement pour la référence à ce genre d'agrégat flou, mais aussi pour la référence pronominale à un procès évoqué par une «simple» CV. Est-ce le fait de poser un contenu propositionnel sur le mode prédicatif qui produit en $\mathrm{M}$ un objet-de-discours correspondant et partant, disponible? Ou est-ce le fait d'y référer qui promeut le contenu au rang d'objet-de-discours ? On trouve une réponse chez Apothéloz, pour qui les opérations de nominalisation (y compris celles réalisées par les pronoms), « établissent au statut de référent, ou d'objetde-discours, un ensemble d'informations qui n'avaient pas antérieurement ce statut discursif» (1995a, p. 144). Selon l'auteur, poser un procès de manière prédicative ne fait pas de lui un objet-de-discours. Ce n'est qu'au moment de l'opération référentielle, qui montre « un changement de niveau ou une condensation de l'information » que le procès devient une entité discursive (ibid.). Ainsi, la nominalisation lexicale ou pronominale marque la véritable consistance de l'objet. Fraurud (1992) se rapproche de cette idée, en invoquant toutefois une conception graduelle de la propriété «être un référent». Elle recourt à une échelle d'hypostatisation, qui rend compte de la capacité à être conçu comme plus ou moins une entité discursive. Lorsqu'un contenu est simplement posé

26 Pour l'analyse d'autres procédés d'indistinction et de confusion référentielle, voir Berrendonner (1990), (1994) et Corminboeuf (2011). 
par une $C V$, il se situerait à l'extrémité minimale de l'échelle, c'est-à-dire qu'il ne constituerait pas un objet bien unifié et bien établi. Par contre, l'opération de référence à un procès, par un pronom neutre par exemple, contribuerait à placer celui-là au degré maximal de l'échelle d'bypostatisation:

Niveau référentiel :

Texte (cf. ex. 1) :

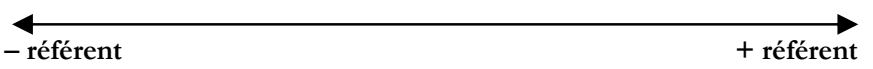

tu as les moustiques qui viennent partout donc ça c'est l'horreur

Ces hypothèses me paraissent néanmoins discutables. Les deux auteurs considèrent qu'un procès désigné constitue un objet, tandis qu'un procès simplement introduit ne possède pas (complètement) ce statut. Si l'usage d'un pointeur pronominal confirme de manière évidente l'existence d'une entité discursive bien établie, pourquoi l'expression d'une $\mathrm{CV}$ n'introduirait-elle pas en $\mathrm{M}$, à la manière d'un $\mathrm{SN}$ indéfini par exemple, un objet-de-discours correspondant? En effet, une position inverse pourrait être de considérer que tout ce à quoi il est possible de référer (par opposition à tout ce à quoi on réfère effectivement) constitue un objet-de-discours. Cela dit, il est possible de référer à quantité d'informations du discours en cours, dont bon nombre de contenus propositionnels (voire tous) ${ }^{27}$. Prenons à titre d'exemple le tout début de l'exemple (19) et modifions-en la deuxième $\mathrm{CV}$, en sorte qu'elle réfère au contenu de la première :

(21) Et alors donc euh, alors c'était super, je te l'assure, parce que [...]

On peut également modifier la troisième $\mathrm{CV}$, de manière à opérer une référence au contenu de la deuxième $\mathrm{CV}$ :

(22) Et alors donc euh, alors c'était super parce que, on était en fai/ en fait avec les Perez, donc ça a nous a beaucoup rassurés [...]

Ces manipulations montrent que pratiquement n'importe quel contenu exprimé par une CV devrait, dans cette hypothèse, être considéré comme un objet-de-discours, de même que n'importe quel aspect de l'interaction en $\operatorname{cours}^{28}$. Ce critère de potentialité à la référence, pour posséder le statut de référent, rencontre donc lui aussi des problèmes de validité : il conduirait selon Fraurud (1992) à une « hypertrophie » de la population de $\mathrm{M}$. On verra ci-après qu'il y a peut-être une autre manière d'envisager la question. Revenons enfin à la proposition de la scalarité du statut d'objet-dediscours (ibid.), qui à mes yeux, se montre peu convaincante: sur quels indices linguistiques se fonde-t-elle? Peut-on vraiment 'être plus ou moins référent'? Cela dit, le concept de gradualité a l'avantage de s'appliquer à la notion d'accessibilité du référent, dont il sera question ci-dessous.

\footnotetext{
${ }^{27}$ Evidemment pas n'importe quand ni n'importe comment, mais dans des conditions particulières qui restent à définir.

28 On peut par exemple référer à n'importe quelle unité linguistique (cf. la notion de deixis textuelle de Lyons, 1990) : Moi aussi j'essaie désespérément (qu'il est lonnnnng ce mot!) d'aimer à la folie Radiohead, mais rien ne se passe! (blog, web)
} 


\subsection{Degré d'activation et degré de validité des objets en $M$}

En effet, la question de l'accessibilité référentielle ou ses notions voisines permettent d'approcher le problème sous un autre angle et d'avancer quelques pistes pour l'étude de la référence aux procès et agrégats. Certains modèles cognitifs se sont attachés à déterminer le rapport entre les expressions référentielles et le statut cognitif des référents visés. L'échelle d'accessibilité (Ariel, 1988), la biérarchie du donné (Gundel et al., 1993) ou encore l'échelle de phoricité (Cornish, 2010 et dans ce volume) s'efforcent de rendre compte du rôle des expressions référentielles dans l'indication de l'accessibilité, du statut cognitif, ou des modalités de récupération des référents: certaines expressions seraient spécialisées dans la mise en focus d'un référent qui était en retrait (par exemple les démonstratifs), tandis que d'autres indiqueraient que le référent est hautement accessible, ou déjà au centre de l'attention des interlocuteurs (par exemple les clitiques). Ces modèles possèdent chacun des caractéristiques distinctives dans le détail desquelles je n'enterai pas ${ }^{29}$, mais tous supposent que les objets-de-discours sont dotés, plus généralement, d'une saillance relative en M. Par analogie à sa définition en analyse perceptive visuelle, on peut considérer la saillance en linguistique comme étant le caractère prépondérant, prégnant de certains éléments : un élément saillant ressort en premier dans la perception d'un message linguistique ou des représentations cognitives du sujet parlant (Landragin, 2004). Divers facteurs peuvent contribuer à rendre saillant un référent en contexte discursif : des facteurs sémantiques, prosodiques, syntaxiques, visuels, la récence d'introduction d'un référent, la structure informationnelle, les intentions et l'attention des interactants, des schémas culturels, des opérations cognitives, etc. (ibid.) En situation de discours, on peut donc supposer qu'un grand nombre d'objets est à la portée des interlocuteurs et que certains d'entre eux sont plus saillants que d'autres à un moment donné ${ }^{30}$. A l'inverse, d'autres référents sont plutôt en retrait. Selon Apothéloz (1995b, p. 169-170), on peut distinguer deux sortes de saillance : une saillance locale, qui n'est pas liée à la construction du sens en cours, mais qui dépend de facteurs «accidentels » de la situation immédiate, tels que la récence d'activation dans le discours (par des moyens verbaux ou non) ou les propriétés perceptives particulières de l'objet, etc. La saillance cognitive, quant à elle, recouvre la centralité d'un objet sur le plan de l'organisation des représentations cognitives en mémoire discursive et a trait à la notion de pertinence :

l'objet le plus saillant cognitivement est alors celui qui occupe la position la plus centrale dans l'univers d'objets considéré : c'est aussi, vraisemblablement, celui dont l'effet organisateur est le plus fort dans cet univers. (ibid., p. 170)

\footnotetext{
${ }^{29}$ Même si je reconnais leur importance, je n'adhère pas complètement à l'un ou l'autre de ces paradigmes : j'admets que le choix d'un type d'expressions référentielles puisse être motivé par le statut cognitif ou l'accessibilité d'un référent, néanmoins, l'intervention d'autres facteurs (interactionnels, planificatoires, pragmatiques, etc.) me parait tout aussi pertinente dans cette phase d'encodage. A ce sujet, je renvoie à Béguelin (1997).

30 cf. aussi par exemple la Théorie du centrage d'attention pour une modélisation des critères qui prédisent le « meilleur » candidat à l'anaphore (Walker et al., 1998), critères toutefois essentiellement linguistiques.
} 
La saillance locale peut être représentée comme une pile d'objets, au sommet de laquelle se tient momentanément le plus saillant d'entre eux, tandis que la saillance cognitive s'apparente davantage à une structure de graphe, ou de treillis, au centre duquel se trouve l'objet le plus saillant au niveau cognitif, qui constitue le nœud vers lequel convergent le plus grand nombre de liens (Apothéloz, ibid.). La manière dont ces deux types de saillance interagissent reste néanmoins assez floue et théorique (ibid., p. 316-317), mais leur distinction peut déjà permettre d'affiner et de nuancer certaines situations. A la suite du Groupe de Fribourg (à par., p. 126-127), j’appellerai la saillance relative d'un objet son degré d'activation momentané en $\mathrm{M}$, qui combine les saillances locale et cognitive. Les opérations de pointages référentiels sont des paramètres importants de cet indicateur, puisqu'elles contribuent à maintenir ou à augmenter l'activation d'un référent.

A côté d'un indice d'activation de saillance associé à chaque objet-de-discours, le Groupe de Fribourg (ibid., p. 248 sqq.) ajoute un indice de validité, autrement dit, un indice signalant le degré d'appartenance de l'objet à la mémoire discursive. Ainsi, c'est moins la nature de référent - tel que suggéré par le concept d'hypostatisation supra que l'appartenance d'un référent à $\mathrm{M}$ qui est graduelle. Parmi les objets-de-discours, certains sont introduits de manière explicite: on n'a aucun doute sur leur validité ; celle-ci est donc maximale. D'autres sont inférés en $\mathrm{M}$ par voie implicite et sont de ce fait le résultat d'un pari probabiliste. Par exemple, si j'aperçois des phares, je peux en inférer l'existence d'une voiture (Berrendonner, 1990). Si cette interprétation est plausible, elle n'en est pas pour autant fiable ${ }^{31}$. Les référents peuvent donc être le résultat d'inférences relevant de degrés de confiance variables.

\subsection{Activation et validité des procès en $M$ : éléments de réflexion}

Tout objet-de-discours est donc doté d'un degré d'activation momentané et d'un degré de validité en $\mathrm{M}$ dont la prise en compte systématique dans les études sur la référence pourrait s'avérer instructive ${ }^{32}$. Précisons qu'un haut degré d'activation n'entraîne pas forcément un haut degré de validité et vice-versa : un objet sous-entendu peut être très saillant, mais en tant qu'objet implicite, il n'est pas complètement valide; inversement, n'importe quel objet explicitement introduit est maximalement valide, mais son degré d'activation varie selon certains facteurs (cf. supra \ 7.3.). Là aussi néanmoins, l'interaction de ces propriétés mérite d'être davantage étudiée.

Revenons-en aux procès : comment envisager l'évolution de ces propriétés dans leur cas? Sont-ils sensibles aux mêmes facteurs que les objets du «1 $1^{\text {er }}$ ordre »? En plus d'être des objets mal connus des scientifiques, les procès, a fortiori les agrégats, apparaissent sous-spécifiés (puisque non catégorisés) et complexes (impliquant souvent

31 Dans la logique Peircienne, ce type de raisonnement s'apparente à une abduction. L'opération consiste, étant admis une implication (P つ Q) et son conséquent (Q), à conclure à l'existence de (P) en « remontant » l'implication :

a) Les ivrognes rayent le boîtier de leur montre en la remontant

b) Or, le boîtier de sa montre est rayé.

c) Donc, cet homme est un ivrogne. (Groupe de Fribourg, à par., p. 244-245)

32 Ces propriétés pourraient par exemple être mises à profit pour distinguer certains types d'anaphore associative, qui reposent sur des inférences de diverses sortes. 
plusieurs actants et relations). Ces caractéristiques les rendent-ils moins saillants ou moins valides? Y a-t-il lieu d'envisager pour eux des échelles distinctes d'activation et de validité ?

A défaut de résoudre ces problèmes complexes, j'évoque ci-dessous un certain nombre de questions et de pistes de recherche :

Avant le pointage :

- Il faut reconnaître que les procès exprimés tour à tour par une CV dans la chaîne parlée sont, en tant qu'objets explicitement introduits, des objets entièrement valides. En ce qui concerne leur degré d'activation, la question s'avère complexe. On peut penser qu'au moment de leur énonciation, ils sont localement saillants, mais qu'à défaut d'anaphorisation subséquente contribuant à les maintenir «en haut de la pile » ou à en faire des centres organisateurs en $\mathrm{M}$, leur degré d'activation diminue rapidement. Reste à déterminer comment mesurer ce degré d'activation. Il conviendrait également de vérifier si les facteurs d'activation sont les mêmes que ceux proposés pour les objets classifiés $^{33}$. Enfin, on peut se demander dans quelle mesure ces procès sont sous-spécifiés : s'ils n'appartiennent pas à une catégorie typique, dont l'indice est l'absence d'une dénomination conventionnelle, ils possèdent néanmoins une dénomination occasionnelle, à savoir l'expression particulière qui les dénote au moment où ils sont introduits (la CV). Au final, du fait qu'ils sont introduits par un descripteur ad hoc, donc qu'ils sont à cette occasion précise "nommés », on peut remettre en cause la nature sous-spécifiée qu'on leur a en premier lieu attribuée.

- Les agrégats sont des objets complexes et mal délimités qui peuvent résulter, par le biais de raisonnements inférentiels, de l'interaction de nombreux procès, de relations et d'indices de diverses sortes, implicites ou non. Ce n'est pas une simple expression qui permet d'introduire de facto un agrégat. En ce sens, ces objets ne sont pas pleinement valides, ni localement saillants. On l'a vu supra, cela n'entraîne pas forcément un degré d'activation très faible : on peut très bien imaginer qu'un locuteur et son allocutaire ont très présente à l'esprit une information sous-entendue. Le calcul du degré d'activation de tels objets implicites se présente comme une tâche encore moins aisée que pour les procès valides et localement saillants. Il y a dès lors des raisons de les considérer comme des objets sous-spécifiés, puisqu'ils ne sont ni explicitement dénommés, ni catégorisés.

Au moment du pointage :

- Un pronom neutre permet de référer à un procès valide comme à un agrégat très complexe dont l'indice de confiance n'est pas maximal. Il serait intéressant d'observer si une différence dans l'usage des pronoms existe à ce niveau-là (e.g. une différence entre clitiques et toniques). En pointant sur un procès, un

\footnotetext{
33 On pourrait par exemple penser au rôle spécifique des schémas d'actions, de la structure narrative, des temps verbaux dans le degré d'activation des procès.
} 
pronom confère à celui-ci une validité maximale, ou la maintient (autrement dit, il confirme son appartenance à $\mathrm{M}$ ), de même qu'il augmente (ou maintient) son degré d'activation momentané. Cela dit, le pronom n'indique aucune catégorisation et en cela, il reste peu parlant sur l'identité et l'extension du procès en question, qui reposent en grande partie sur d'autres indices interprétatifs. Cela montre que la référence pronominale aux procès au sens large joue avec une certaine «zone d'incertitude »: tout en confirmant l'existence et la saillance d'un procès, elle maintient l'incertitude autour de ses contours. Et plutôt que considérer cette incertitude comme gênante, on peut au contraire la regarder comme une disposition productive en conversation, autorisant de manière économique une malléabilité référentielle (cf. la « chaîne approximative » de l'exemple (18)) que d'autres types d'expressions référentielles plus spécifiées ne connaissent pas.

\section{Conclusions}

Ce papier s'interrompt en plein milieu de réflexions et d'interrogations qui, elles, poursuivront leur chemin dans la problématique complexe de la référence. A l'issue de cette étude, j’ai suggéré quelques points de discussion qui mériteraient à mes yeux davantage d'attention. La référence anaphorique aux procès est en effet loin d'avoir levé tous ses voiles, notamment dans des contextes de français parlé, qui rendent difficiles l'application des outils d'analyse issus d'une tradition logico-philosophique. En partant de l'observation des données, on s'aperçoit que dans de nombreux emplois, les pronoms neutres se montrent insensibles aux distinctions sémantiques usuelles. Au contraire, leur sous-spécification sémantique intrinsèque semble leur conférer une flexibilité référentielle les rendant productifs et faciles d'emploi pour la désignation d'objets pas toujours bien déterminés. Ce genre de référence pose inévitablement la question des modalités d'existence des procès et de leur statut discursif à différents moments de l'évolution de la mémoire discursive. Pour amorcer une réflexion autour de cette question, j’ai privilégié le recours aux notions d'activation et de validité référentielles. Ces notions me paraissent en effet susceptibles d'apporter du sang neuf aux études sur les procès, mais aussi sur les objets-de-discours en général. Ces conclusions sont aussi l'occasion de relever les limites de l'étude de faits discursifs : je me suis à plusieurs reprises inscrite dans une approche qui conçoit le discours comme un complexe pluricodique, i.e. qui relève de sources d'informations sémiotiquement variées ${ }^{34}$. Il s'agit alors de ne pas perdre de vue que les indices à disposition, auxquels se limitent la plupart des études en linguistique, dont celle-ci, relèvent uniquement du segmental et sont de ce fait nécessairement parcellaires par rapport à la dimension complexe du discours.

\footnotetext{
34 Cela dit, les représentations en mémoire discursive sont sémiotiquement indifférenciées, i.e. elles ont toutes le format d'objets-de-discours (Groupe de Fribourg, à par., p. 23)
} 


\section{Bibliographie}

AMsiLi, P. \& Roussarie, D. (2007), «Interpréter les pronoms phrastiques », Modèles linguistiques, n56, p. $83-112$.

ApothÉLOZ, D. (1995a), «Nominalisations, référents clandestins et anaphores atypiques », Tranel, $\mathrm{n}^{\circ} 23$, p. 143-173.

ApothÉLOZ, D. (1995b), Rôle et fonctionnement de l'anaphore dans la dynamique textuelle, Genève, Droz.

AsHer, N. (1993), Reference to Abstract Objects in Discourse, Dordrecht, Kluwer.

BÉGUELIN, M.-J. (1997), «Stratégies référentielles et variation », Langue française, n 115, p. 101 110.

Berrendonner, A. (1990), «Attracteurs », Cabiers de linguistique française, n¹1, p. 149-158.

BERRENDONNER, A. (1994), «Anaphores confuses et objets indiscrets » in Schnedecker, C. et al. (éds.), L'anaphore associative. Aspects linguistiques, psycholinguistiques et automatiques, Paris, Klincksieck, p. 209-230.

BerRendonNer, A. (2008), « Pour une praxéologie des parenthèses », Verbum, XXX, p. 5-23.

BERRENDONNER \& [REICHLER]-BÉGUELIN (1996), «De quelques adjectifs à rendement anaphorique : premier, dernier, autre », Studi Italiani di Linguistica Teorica e Applicata, n²5, 3, p. $475-502$.

Blanche-Benveniste, C. et al. (2002), Choix de textes de français parlé : 36 extraits, Paris, H. Champion.

CONSTEn, M., KNEES, M., \& SCHWARZ-FriesEL, M. (2007), «The function of complex anaphors in texts. Evidence from corpus studies and ontological considerations ", in SchwarzFriesel, M. et al. (éds), Anaphors in Text. Cognitive, formal and applied approaches to anaphoric reference, Amsterdam/Philadelphia, John Benjamins Publishing Company, p. 81-102.

CorbetT, G.G. (1991), Gender, Cambridge, Cambridge University Press.

CORBLIN, F. (1994), "Existe-t-il un ça impersonnel en français?», L'Information grammaticale, $\mathrm{n}^{\circ} 62$, p. $45-47$.

Corminboeuf, G. (2011), « Dualités », in Corminboeuf, G. \& Béguelin, M.-J. (éds.), Du système linguistique aux actions langagières. Mélanges en l'honneur d'Alain Berrendonner, Bruxelles, De Boeck-Duculot, p, 473-486.

CORNISH, F. (1991), «Non-discrete reference, discourse construction, and the French neuter clitic pronouns ", Journal of French Language Studies, $n^{\circ} 1$, p. 123-138.

CORNISH, F. (1999), Anaphora, Discourse, and Understanding: Evidence from English and French, Oxford, Clarendon.

CORNISH, F. (2001), "L'anaphore pronominale indirecte: une question de focus ", in De Mulder et al. (éds.), L'anaphore pronominale et nominale, Amsterdam, Rodopi, p. 1-25..

CORNISH, F. (2010), « Anaphora: Text-based or discourse-dependent? Functionalist vs. formalist accounts », Functions of Language, vol. 17, n², p. 207-241. 
DesCOMBES DÉNERVAUd, M. \& JeSPERSEN, J. (1992), «L'anaphore conceptuelle dans l'argumentation écrite ", Pratiques, n 73 , p. 79-95.

DIK, S.C. (1997), The Theory of Functional Grammar. Part 1: The Structure of the Clause, Dodrecht, Foris.

DuRAND, J. et al. (2002), «La phonologie du français contemporain: usages, variétés et structure ", in Pusch, C. \& Raible, W. (éds.), Romanistische Korpuslinguistik- Korpora und gesprochene Sprache/Romance Corpus Linguistics - Corpora and Spoken Language, Tübingen, Gunter Narr Verlag, p. 93-106.

DURAND, J. et al. (2009), «Le projet PFC: une source de données primaires structurées », in Durand, J. et al. (éds.), Phonologie, variation et accents du français, Paris, Hermès. p. 19-61.

Fraurud, K. (1992), Processing Noun Phrases in Natural Discourse, PhD dissertation, Stockholm University.

FreGE, G. (1892/1971), Écrits logiques et philosophiques, Paris, Seuil.

Halliday, M. \& Hasan, R. (1976), Cohesion in English, London, Longman.

Grevisse, M. \& Goosse, A. (2007) (14e éd.), Le bon usage, Bruxelles, De Boeck Duculot.

Groupe De Fribourg (à par.), Grammaire de la période I, Berne, Peter Lang.

Gundel, J.K., Hegarty, M. \& Borthen, K. (2003), « Cognitive Status, Information Structure, and Pronominal Reference to Clausally Introduced Entities ", Journal of Logic, Language and Information, n²12, p. 281-199.

Johnson-Laird, P.N. (1983), Mental Models :Towards a Cognitive Science of Language, Inference, and Consciousness, Cambridge, Cambridge University Press.

KLEIBER, G. (1994a), Nominales. Essais de sémantique référentielle, Paris, Armand Colin.

KLEIBER, G. (1994b), Anaphores et pronoms, Louvain-La-Neuve, Duculot.

KLEIBER, G. (1997), «Sens, référence et existence : que faire de l'extra-linguistique ?», Langages, n'127, p. 9-37.

KRIPKE, S. (1959), «A Completeness Theorem in Modal Logic », Journal of Symbolic Logic, $\mathrm{n}^{\circ} 24$, p. $1-15$

LANDragin, F. (2004), "Saillance physique et saillance cognitive», CORELA, vol. 2, n²2. http://corela.edel.univ-poitiers.fr/index.php?id=603, consulté le 10.01.2012.

LYONS, J. (1977), Semantics, Cambridge, CUP.

LYONS, J. (1990), Sémantique linguistique, Paris, Larousse.

MAILLARD, M. (1994), "Concurrence et complémentarité de il et ça devant les prédicats impersonnels en français contemporain », L'information grammaticale, nº2, p. 48-52.

Prince, E. (1981), «Toward a Taxonomy of Given-New Information», in Cole, P. (éd.), Radical Pragmatics, New York, Academic Press, p. 223-256.

[Reichler-]BÉguelin, M.-J. (1988), «Anaphore, cataphore et mémoire discursive », Pratiques, $\mathrm{n}^{\circ} 57$, p. $15-43$.

RiEGEL M. et al. (2009), Grammaire méthodique du français, Paris, PUF. 
Laure Anne Johnsen

Rosch, E. et al. (1976), «Basic objects in natural categories", Cognitive Psychology, nº, p. $382-$ 439.

Vendler, Z. (1967), Linguistics in Philosophy, Ithaca NY, Cornell University Press.

VENDler, Z. (1970), «Say what you think », in Cowan, J.L. (éd.), Studies in Thought and Language, Tucson Arizona, The University of Arizona Press, p. 79-97.

Walker, M.A., Joshi, A.K. \& Prince, E. (éds) (1998), Centering Theory in Discourse, Oxford, Clarendon Press.

WEBBER, B. (1991), «Structure and ostension in the interpretation of discourse deixis», Language and Cognitive Processes, nº, p. 107-135. 